\title{
MINIREVIEW: ADDRESSING THE RETRO-MICHAEL INSTABILITY OF MALEIMIDE BIOCONJUGATES
}

This minireview explores published strategies to counteract the retro-Michael instability of the thiosuccinimide linkage, a limiting factor of the efficacy and safety of antibody-drug conjugates. 
We, the authors, confirm here that all work reported herein is our own work, or the source of the work has been indicated.

Vijay Chudasama

Calise Bahou

Peter Szijj

Table of Contents

Declaration

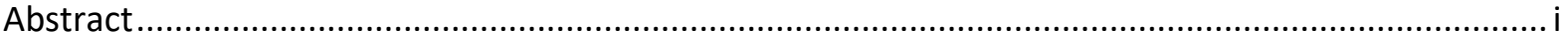

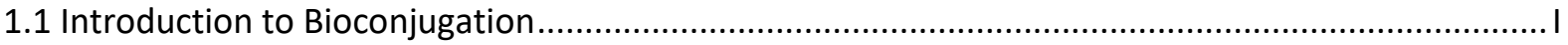

1.2 Introduction to the concept of "Self-hydrolysing maleimides" ......................................................I

2. Methods to increase the rate of thiosuccinimide hydrolysis ......................................................... II

3. Alternative solutions to retro-Michael deconjugation ....................................................................

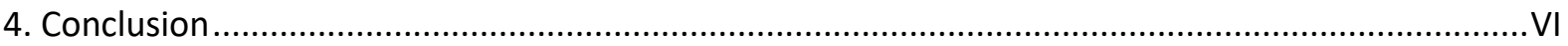

Acknowledgements / Declaration of Contributions ............................................................................ VII

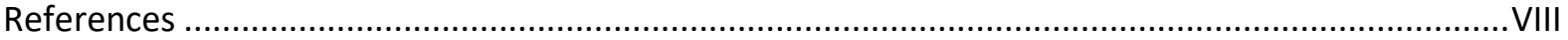

Abstract

Bioconjugation, the modification of biological macromolecules such as proteins, is an up and coming area in the field of chemical biology. Antibody-drug conjugates (ADCs), combining the antigenselectivity of natural antibodies with the cytotoxic potency of small molecule drugs, are a powerful therapeutic technology. Four such constructs are currently on the market for cancer therapy. However, the conjugation methodology employed in these therapeutics is far from ideal. Herein we provide an overview on methods that attempt to increase the safety and efficacy of ADCs via "selfhydrolysing maleimides" or by improving the stability of maleimide-conjugates by other means. We find that some very promising reagents have been reported, however the mechanism by which these reagents act is not clear, thus limiting rational design.

Keywords: Bioconjugation, Organic Chemistry, Chemical Biology, Protein modification 
Protein bioconjugation has emerged in recent years as an important area of research within the field of chemical biology. Exploiting the high fidelity of protein-protein interactions, these constructs can have a variety of medicinal and biotechnological applications, including protein half-life extension [1], emulation of post-translational modification [2] and exploration of new treatment options for cancer [3].

In the area of cancer research, an emergent class of new therapeutics are antibody-drug conjugates (ADCs). These constructs combine the exquisite antigen-selectivity of antibodies with the potent cytotoxic properties of small-molecule drugs. The method provides an edge in efficacy over unmodified antibody therapy alone, whilst reducing off-site toxicity (and thus side-effects) compared to conventional small molecule chemotherapy enabling the use of more powerful drugs [4-6].

There are currently four ADCs approved for use by the food and drug administration (FDA), Brentuximab vedotin $\left(\right.$ Adcetris $\left.^{\circledR}\right)$, Trastuzumab emtansine (Kadcyla $\left.{ }^{\circledR}\right)$, Inotuzumab Ozogamicin (Besponsa ${ }^{\circledR}$ ), and Gemtuzumab Ozogamicin (Mylotarg ${ }^{\circledast}$ ) with more than 60 currently under clinical evaluation [7]. The lessons learned from the development of these ADCs, and many failed ADCs, highlight the importance of optimising the attachment method of drug to protein. Kadcyla ${ }^{\circledR}$, Besponsa ${ }^{\circledR}$ and Mylotarg ${ }^{\circledR}$ are constructed via modifying the antibody's surface accessible lysine residues. However, as lysine residues are abundant in proteins (ca. $3.6 \%$ in terrestrial organisms) [8], this method leads to highly heterogeneous conjugates when targeting typical drug to antibody ratios (DARs) of 2-4 [9-11]. Cysteine modification, as seen with Adcetris ${ }^{\circledR}$, has gained popularity in recent years due to the high nucleophilicity, selectivity towards soft electrophiles, and low natural abundance of the sulfhydryl group-bearing amino acid residue in naturally occurring proteins (ca. $0.15 \%$ in terrestrial organisms) $[8,12]$. In Adcetris ${ }^{\circledR}$, the 4 accessible interchain disulfide bonds are partially reduced to liberate cysteines that can be site-selectively modified. Whilst this still results in heterogeneous conjugates when targeting typical DARs (partial reduction is not selective for the hinge or Fab interchain disulfides), it is far less heterogeneous than modifying lysine residues.

Typically, maleimides have been used extensively for cysteine modification as they react rapidly and selectively with thiols. Functionalising maleimides by adding substituents on the $\mathrm{N}$-atom is also facile. In the instance of Adcetris ${ }^{\circledR}$, and many other ADCs under evaluation, a maleimide linkage is used to modify the solvent accessible cysteines liberated from partial reduction of disulfide linkages on an IgG1 antibody. However, it has recently come to light that the thioether linkage undergoes deconjugation through a retro-Michael pathway, leading to loss of cargo and reduction in efficacy. The maleimide-cargo conjugate can then be bound to other plasma thiols (e.g. human serum albumin) leading to off-site toxicity and reduced efficacy (Scheme 1a) [13-15].

A slew of methods has been developed to circumvent this problem of instability inherent in maleimide-cysteine conjugation. Of great interest in the field is the concept of "self-hydrolysing maleimide" reagents. These compounds undergo rapid hydrolysis to the corresponding succinamic acid post-conjugation, thus eliminating the retro-Michael deconjugation pathway and resulting in more effective/stable bioconjugates [16-23]. Alternatively, researchers have employed distinct thiol reactive groups, [24-27] or tweaked the traditional maleimide scaffold to obviate the issue, e.g. adding leaving groups to the double bond [28] or using exocyclic olefinic maleimides [29]. Herein a review of the area will be given. 
Although it has been known for many years that on hydrolysis, maleimide reagents lose their reactivity towards thiol-bearing functionalities, $[30,31]$ it was only recently that Shen and co-workers proposed that post-conjugation hydrolysis increases the stability of thiol-maleimide conjugates [13]. Using antibodies with cysteines engineered in various unnatural positions, they showed that local protein micro-environment has a powerful effect on the stability of the bioconjugate. They proposed that local positively charged residues promote rapid thiosuccinimide ring hydrolysis, and thus limit thiol-exchange mediated loss of cargo (Scheme $1 \mathrm{~b}$ ). In a preliminary safety study, they also show that more stable conjugates lead to less liver toxicity in rats.

a)

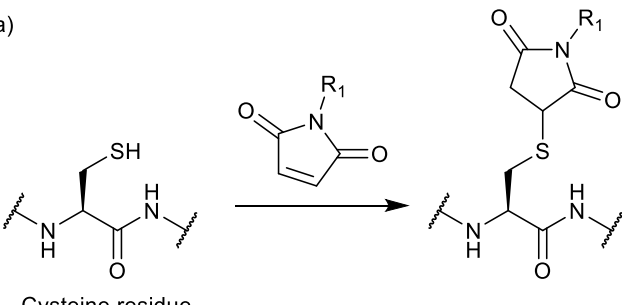<smiles>CCNC(=O)C(CS)NC(C)CC(C)C</smiles>

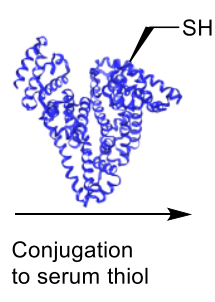

Cysteine residue

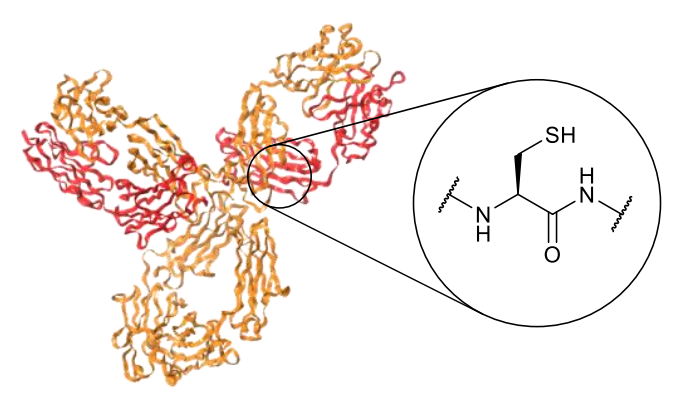

b)<smiles>[R3]N1C(=O)C=CC1=O</smiles>
Cysteine residue

\begin{tabular}{|l|l|}
\hline $\begin{array}{l}\text { Compound } \\
\text { indentifier }\end{array}$ & 1 \\
\hline $\mathrm{R}_{1}$ group & \\
\hline
\end{tabular}

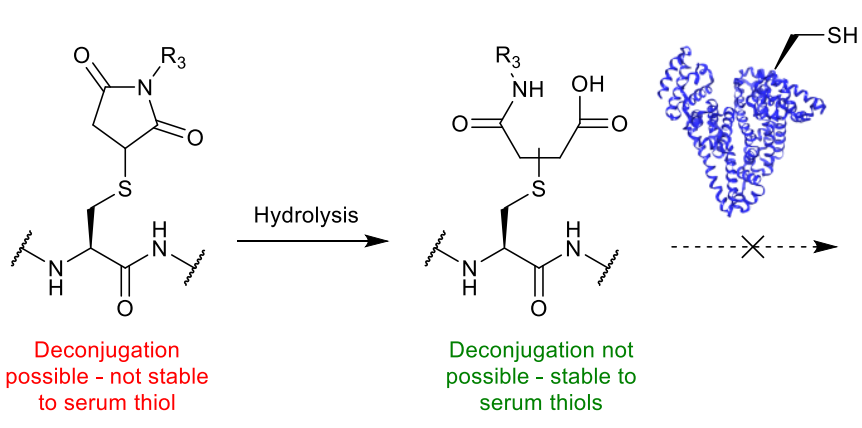

\begin{tabular}{|l|c|c|c|c|c|}
\hline $\begin{array}{l}\text { Compound } \\
\text { indentifier }\end{array}$ & 2 & 3 & 4 & 5 & 8 \\
\hline \multirow{2}{*}{$\mathrm{R}_{3}$ group } & & & 4
\end{tabular}

Scheme 1

Hydrolysis of maleimide linkers confers stability to retro-Michael deconjugation. Crystal structures of antibody [37] and human serum albumin [38] adapted from the RSC PDB using Maestro.

In the years following the Shen et al. paper [13] in 2012, the development of methods to create maleimide reagents that hydrolyse rapidly after conjugation, without the requirement for a specific local microenvironment, were explored.

2. Methods to increase the rate of thiosuccinimide hydrolysis 
In 2014, Tumey and co-workers observed that an ADC prepared via maleimide-thiol conjugation using a maleimide reagent bearing six polyethylene glycol (PEG) units (Scheme 1, maleimide 2) hydrolysed whilst in a neutral storage buffer. In contrast, an analogous ADC constructed using a maleimidocaproyl linker (Scheme 1, maleimide 1) showed no hydrolysis. They proposed that coordination of water to the proximal PEG-oxygen of the linker was responsible for the increased rate of hydrolysis. The rate of hydrolysis of the succinimides in the ADC created with PEG maleimide 2 was evaluated at $37^{\circ} \mathrm{C}$ and shown to progress to complete hydrolyse by mass spectrometry over $14 \mathrm{~h}$ in borate buffered saline (BBS) at pH 9.2. However, in PBS at pH 7.4, only $30 \%$ hydrolysis was observed over $16 \mathrm{~h}$. Moreover, and perhaps as expected, on comparing the unhydrolysed maleimidocaproyl-bearing ADCs to the hydrolysed maleimide-PEG derived ADCs, the latter showed improved plasma half-lives and efficacies in mice [16].

Also in 2014, Lyon et al. [17] showed that a maleimide reagent incorporating an amino group at a specific position (Scheme 1, maleimide 3) underwent rapid hydrolysis when unconjugated. They proposed that the reported half-life of around 25 mins under mild conditions ( $\mathrm{pH} 7.4$ and $22{ }^{\circ} \mathrm{C}$ ) was facilitated by intramolecular base catalysis. The stability of ADCs prepared with "self-hydrolysing" maleimide $\mathbf{3}$ and maleimide $\mathbf{1}$ was appraised. The hydrolysis half-life of the bioconjugate bearing compound 3 was estimated to be $2.6 \mathrm{~h}$ and $2.0 \mathrm{~h}$ for the light and heavy chain, respectively. In contrast, no hydrolysis was observed at $24 \mathrm{~h}$ for the ADC bearing the maleimidocaproyl linker 1 . Over two weeks in an $\mathrm{N}$-acetyl cysteine containing buffer at $\mathrm{pH} 8$ and $37^{\circ} \mathrm{C}$ their "self-hydrolysing" $A D C$ showed no measurable drug loss, while the control ADC lost $c a$. half of its drug component. The comparison showed similar results in rat plasma and in rats in vivo. The group also showed that the more stable ADC led to lower off-site bone marrow toxicity in rats as well as substantially increased anti-tumour activity.

A study of thiosuccinimide hydrolysis by Fontaine et al. offered a different explanation of the kinetics of the aforementioned self-hydrolysing reactions [18]. They compared the rates of hydrolysis and thiol-exchange for around twenty maleimide based conjugates containing varyingly powerful electron withdrawing groups (EWGs) and observed a linear correlation between Taft $\sigma^{*}$ polar substituent constants and hydrolysis rates. They proposed that the effect observed by Lyon et al. was due to the electron withdrawing effect of the protonated amino group and not due to base catalysis. They showed that $4^{\circ}$ amines, which cannot act as base catalysts but have large positive $\sigma^{*}$, also increase the rate of hydrolysis at a comparable rate. They proposed that the beneficial effect of PEG spacers found by Tumey et al. also arose from an electron withdrawing effect, rather than from co-ordination. They also found that EDGs (such as alkyl and carboxylate) slowed hydrolysis, further substantiating their claims.

Furthermore, they calculated the sensitivities to electron withdrawal of both the hydrolysis reaction and the thiol-exchange reaction by invoking the Taft polar sensitivity factor $\rho^{*}$. The $\rho^{*}$ for the former reaction was found to be 1.0 while the $\rho^{*}$ for the second process was calculated as 0.48 , indicating that succinimide hydrolysis has a higher sensitivity to electron withdrawing groups.

In line with these findings Christie et al. [19] reported that unconjugated $\mathrm{N}$-phenyl maleimides (Scheme 1 maleimide 4) hydrolyse $\sim 5.5$ and react with thiols $\sim 2.5$ times faster than $N$-alkyl maleimides at physiological $\mathrm{pH}$, with the half-life of the former process being $\mathrm{ca}$. 55 mins. Adding an electron withdrawing fluorine atom to the phenyl ring decreases the $t_{1 / 2}$ to 28 mins (Scheme 1 , maleimide 5). Slower hydrolysis rates were observed post-conjugation; $\mathrm{N}$-alkyl thiosuccinimides having a $27 \mathrm{~h}$ half-life compared to $1.5 \mathrm{~h}$ for the $\mathrm{N}$-aryl variant and $0.7 \mathrm{~h}$ for the $\mathrm{N}$-fluorophenyl conjugate at $\mathrm{pH} 7.4,37^{\circ} \mathrm{C}$. 
On incubation in PBS containing $\beta$-mercaptoethanol or in mouse serum, the stability of ADCs prepared with $N$-aryl maleimides was shown to be substantially better than those prepared with $\mathrm{N}$-alkyl maleimides. The former retained $90-100 \%$ conjugation, in both cases over a $200 \mathrm{~h}$ period, with the latter suffering from $60-70 \%$ deconjugation. Perhaps as expected, increased efficacy was observed for the more stable conjugate in a cell killing assay [19].

Following these findings, a method combining the electron withdrawing ability of a phenyl group and the activity (acid catalysis as claimed by the publishing group) of an amino group was reported [20]. The $\mathrm{N}$-acetyl cysteine adduct of o-aminoethyl- phenylmaleimide (Scheme 2, maleimide 6) was shown to completely hydrolyse over a $2 \mathrm{~h}$ period at room temperature and $\mathrm{pH} 7$ post-conjugation to $\mathrm{N}$-acetyl cysteine. To eliminate intramolecular macrocyclisation between the amino group and the carboxylate group of the thiosuccinimide ring, an $\mathrm{N}$-isopropyl variant of the linker was prepared. The $t_{1 / 2}$ of the $\mathrm{N}$-acetyl cysteine conjugate of this linker was found to be 20 mins at room temperature at $\mathrm{pH} 7$, while under the same conditions the corresponding conjugate of $\mathrm{N}$-aminoethyl maleimide was observed to have a half-life of $3.6 \mathrm{~h}$. After demonstrating the cysteine selectivity and rapid hydrolysis of their scaffold on proteins, a method to increase the shelf-life of the linker molecule was approached. A photocleavable 6-nitroveraltryl-oxycarbonyl (NVOC) group was added to the amino group of the reagent (Scheme 2, maleimide 7), thus masking its catalytic activity. A major improvement in shelf-life was observed. To test the scaffold, a bioconjugation reaction with cysteine-containing tripeptide glutathione and $\mathrm{N}$-acetyl cysteine was carried out on ice, to generate the bioconjugate in 10 mins. The constructs were then irradiated with UV light for 50 mins, and complete conversion to the ring-opened bioconjugate was observed by HPLC analysis.

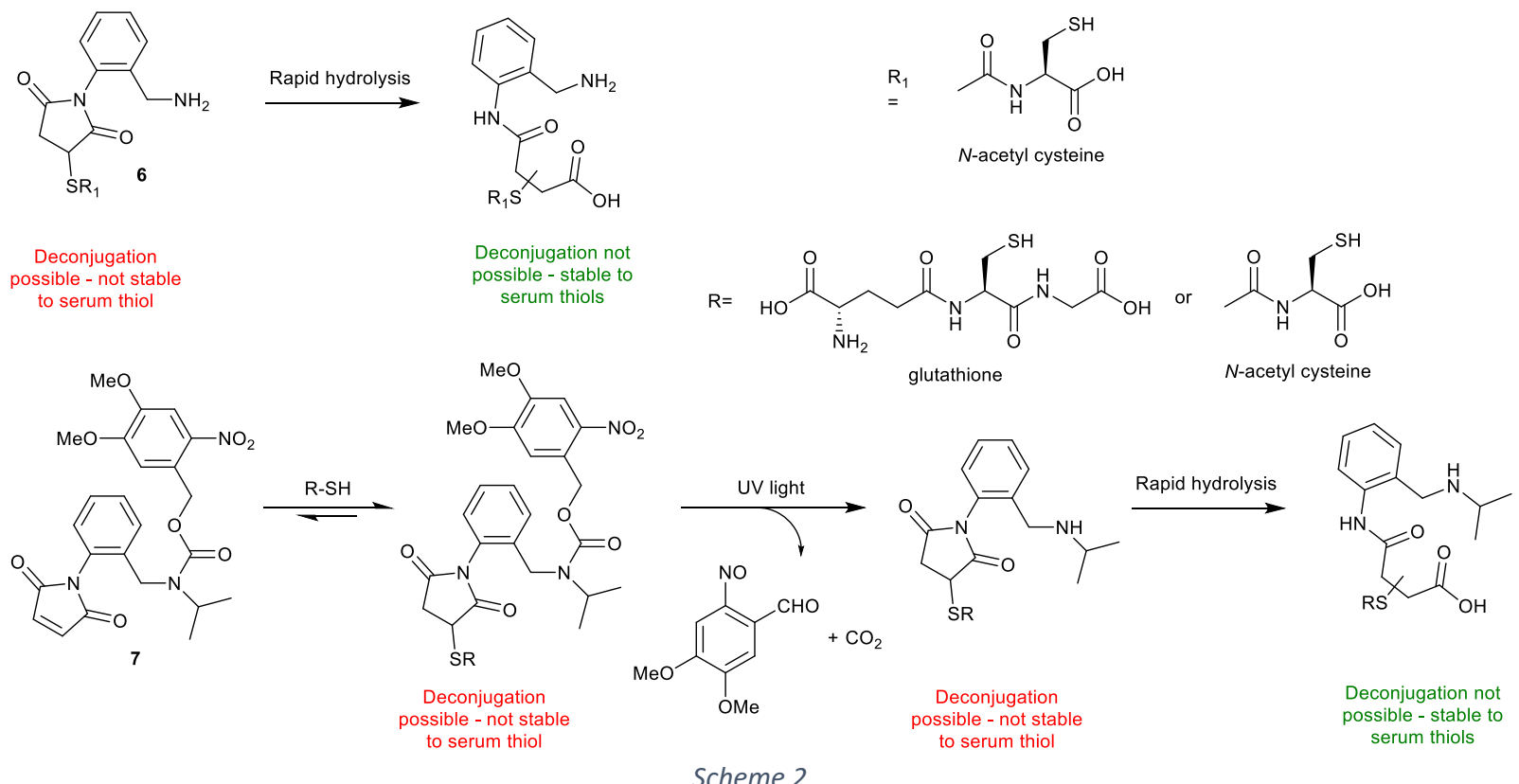

Kalia et al. method for UV light triggered maleimide self-hydrolysis.

A popular method for coupling thiols to amines is the succinimidyl 4-( $\mathrm{N}$-maleimidomethyl)cyclohexane-1-carboxylate (SMCC) heterobifunctional reagent, however, the thiosuccinimide linkage of this has been shown to be prone to thiol-exchange [21]. A study undertaken to improve SMCC solubility found that by replacing the cyclohexyl group with a 1,3-dioxane ring, the rate of thiosuccinimide hydrolysis increased. The dioxane-bearing probe hydrolysed completely over $29 \mathrm{~h}$, while only trace amounts of the hydrolysed cyclohexyl probe were observed via a fluorescence resonance energy transfer (FRET) assay [22]. 
Following on from these findings, Tobaldi and co-workers investigated the use of a reagent with a dioxolane ring attached to a maleimide ring via a methylene bridge (Scheme 1, compound 8) for use in bioconjugation [23]. In PBS (10\% DMSO), at $37^{\circ} \mathrm{C}$ and pH 7.4, the dye-conjugate of this molecule showed complete ring hydrolysis in less than $20 \mathrm{~h}$. Surprisingly, in human plasma, at the same temperature, the hydrolysis was considerably slower, showing close to $65 \%$ hydrolysis over $30 \mathrm{~h}$. Next, thiol-exchange rates were measured, and the construct was found to undergo no thiolexchange with albumin when hydrolysed prior to incubation, and only modest thiol-exchange when not pre-hydrolysed, plateauing at $<30 \%$ after 7 days. The authors offer no mechanistic explanation for the increased rate of hydrolysis for the dioxo linkers, but both the water-to-oxygen co-ordination theory of Tumey et al. [16] and the electron withdrawing effect based explanation of Fontaine et al. [18] could be invoked.

\section{Alternative solutions to retro-Michael deconjugation}

While the aforementioned self-hydrolysing maleimides are a promising route towards preventing retro-Michael deconjugation, it should be noted that other strategies exist to prevent this altogther. A simple and inexpensive solution is to incorporate a leaving group (i.e. a bromine atom) on the vinylic bond of the maleimide scaffold, forming an unsaturated thio-succinimide linkage post conjugation that is mechanistically stable to the retro-Michael process [28]. However, hydrolysis is still considered essential for this strategy as the unsaturated thio-succinimide moiety is susceptible to additional thiol exchange, resulting in suboptimal in vivo stability without hydrolysis.

Most recently, reported by Forte et al. [32], substituted maleimide conjugation can be considered an intricate balance between rate of cysteine conjugation and pre-conjugation hydrolysis (ideally quick and slow respectively). Forte et al. also provided evidence to suggest varying the leaving group on the maleimide affects both the conjugation and hydrolysis rates with diiodomaleimides presenting the most ideal profile of rapid conjugation and slow pre-conjugation hydrolysis [32]. It is also noted from research presented from Morais et al. [33] that varying the dibromo maleimide $\mathrm{N}$-directed handle had a direct influence on maleimide hydrolysis. This work highlighted functional groups (e.g. aromatics) that exhibit a pre-conjugation hydrolysis rate similar to the typical cysteine conjugation rate, resulting in low conjugation yields (Scheme 3a). A similar class of molecules called bromopyridazinediones exploit the same principles as substituted maleimides, but pose a hydrolytically stable alternative for cysteine functionalisation [34]. Furthermore, the conjugated pyridazinedione scaffold is stable in vivo (blood) with no further hydrolysis required (Scheme 3b) $[35,36]$. It is noted however, that this moiety is less reactive towards cysteine than the maleimide scaffold, and so quantitative formation of the bioconjugate can take longer (up to $16 \mathrm{~h}$ ).

Additionally, a strategy based on exocyclic olefinic maleimides has been reported, [29] where having the double bond exocyclic to the maleimide ring offers a substantial improvement in the stability of the conjugate towards thiol-exchange (Scheme 3c). The authors of the study attribute this increased stability to reduced mesomeric stabilisation of their scaffold compared to traditional endocyclic maleimides. The synthesis of a wide range of such compounds has been reported through a Wittiglike reaction offering good yields of exocyclic olefinic maleimides. 
a)

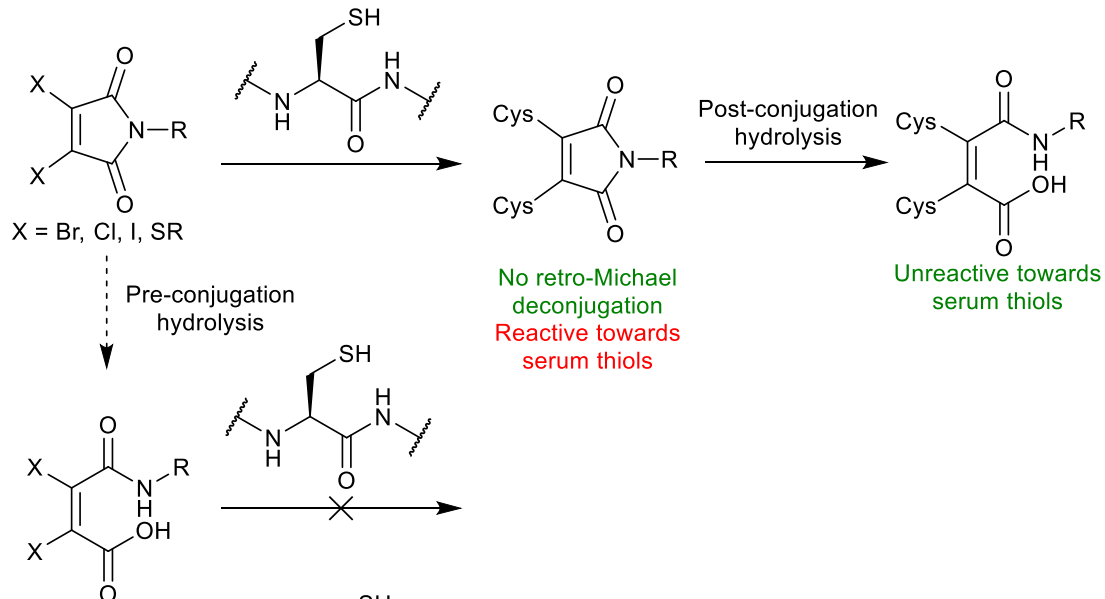

b)
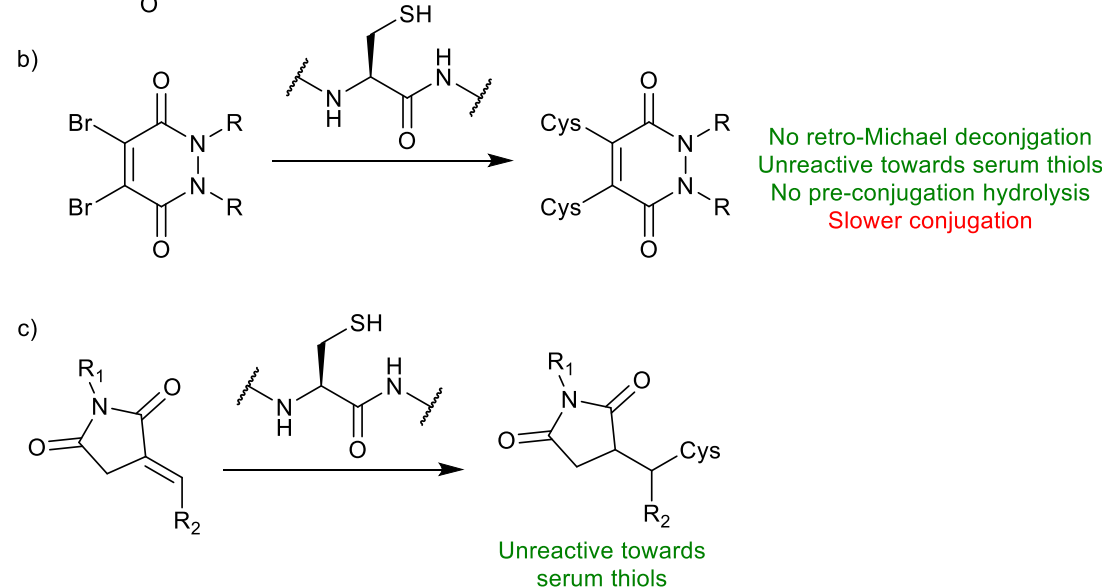

Scheme 3

Non-classical maleimide-based solution to the retro-Michael deconjugation mediated instability of the thiosuccinimide linkage. a) "Next generation" maleimides. b) Bromopyridazinediones. c) Exocyclic olefinic maleimides.

\section{Conclusion}

To conclude, several promising solutions to the retro-Michael instability of the thiosuccinimide linkage have been proposed. Unfortunately, as no study comparing these different methods exists to date, it is difficult to make a substantive/conclusive statement about the relative merit of these approaches. It would be interesting and beneficial to the scientific community for such a study to be carried out, potentially incorporating an even wider array of bioconjugation techniques. This would allow identification of the most promising candidates, so the scientific community may focus its efforts on these. As of now, based on available data, no clear "best approach" can be identified. All methods mentioned above have their advantages and drawbacks, and the "best fitting" approach for now at least must be identified on a case-by-case basis.

Nonetheless, whilst multiple approaches to self-hydrolysis have been reported (Table 1), it can be argued that the photocaged maleimide linker reported by Kalia et al. (maleimide 6) can be considered as an evolution of the concept. It combines multiple positive effects on hydrolysis rate as reported above (i.e. phenyl ring and amino group) whilst also obviating the issue that reagents that hydrolyse fast post-conjugation can also be expected to hydrolyse fast pre-conjugation, which is detrimental to efficiency. This reagent has an extended shelf-life and retains the potential for a rapid rate of hydrolysis, the exact timing of which can be controlled by UV irradiation [20]. In terms of mechanistic explanations for the effect of self-hydrolysis, no consensus has been reached, with Lyon and co-workers suggesting a base-catalysed effect of the proximal amino group, [17] while Fontaine 
et al. convincingly argued that the inductive electron withdrawing effect of the protonated amino group is responsible for the increased rate of hydrolysis [18]. Christie and co-workers have argued that the mesomeric electron withdrawing effect of the phenyl group is at play in their method, [19] and Kalia et al. proposed that their aminoethyl phenyl maleimide reagent combines both the effects of mesomeric electron withdrawal and acid catalysis. To rationally design even more efficient reagents, a firm mechanistic understanding of the phenomenon would be required. Thus, experiments designed to increase our understanding of the mechanism of thiosuccinimide hydrolysis would be beneficial. However, even in their current state, these reagents undoubtedly provide an interesting solution to the thiosuccinimide instability problem, and the recent "triggered" hydrolysis method could pave the way for next generation thiol-maleimide conjugates.

Table 1

Summary of self-hydrolysing maleimide methods reported to-date.

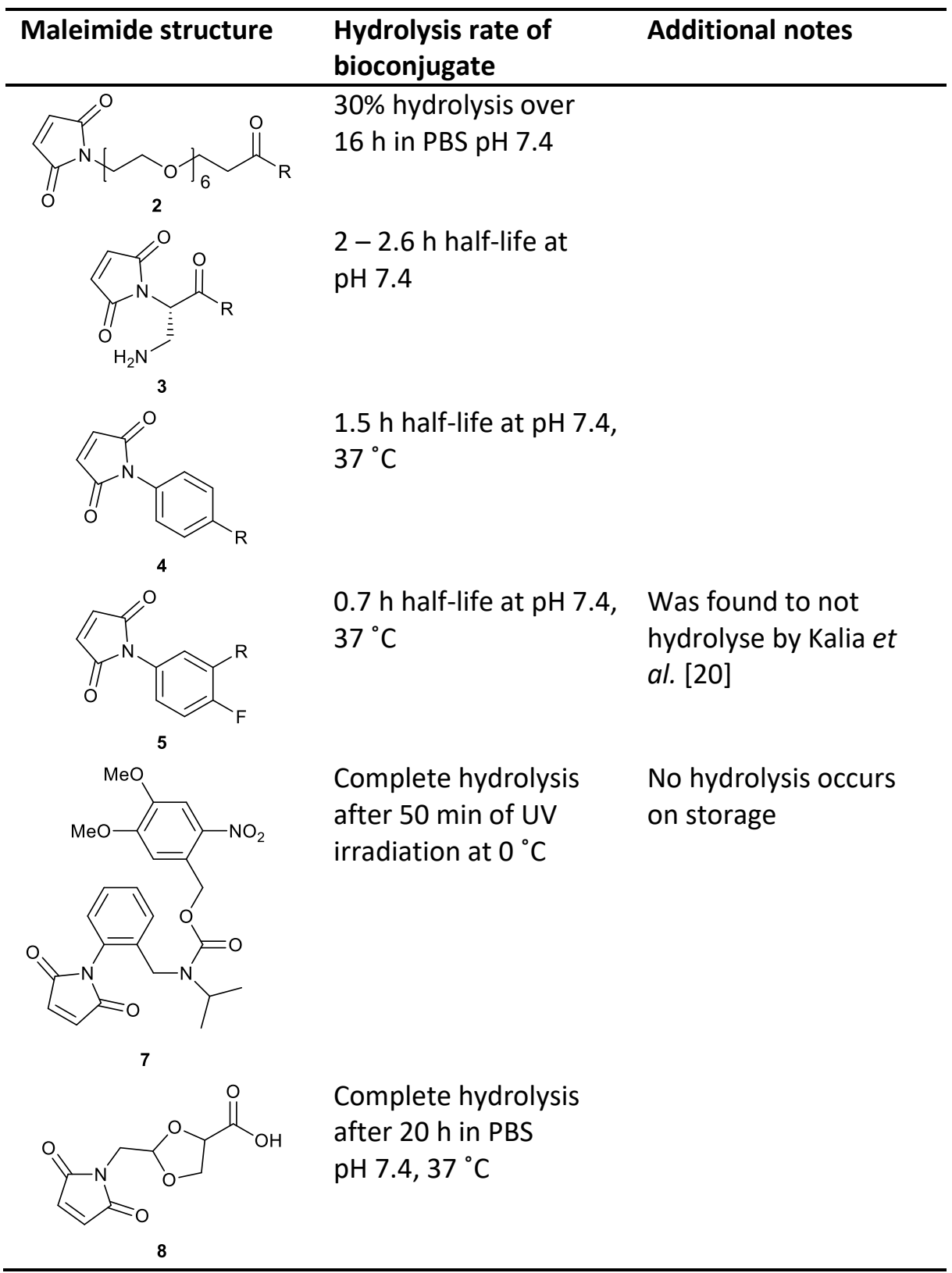


The authors would like to thank members of the Chudasama, Baker and Sheppard research groups for many an insightful discussion. P.S. would like to thank the Wellcome Trust for the generous funding it provides. C.B. would like to gratefully acknowledge the EPSRC (CASE Award with LifeArc, 173621) for funding. P.S., C.B. and V.C. co-wrote the review and co-analysed the literature.

References

[1] Brocchini S, Badescu G, Bryant P, Swierkosz J, Khayrzad F, Pawlisz E, et al. A new reagent for stable thiol specific conjugation. Bioconjug Chem 2013;25:460-9.

[2] Walsh CT, Garneau-Tsodikova S, Gatto GJ. Protein posttranslational modifications: The chemistry of proteome diversifications. Angew Chemie - Int Ed 2005;44:7342-72.

[3] Verma S, Miles D, Gianni L, Krop IE, Welslau M, Baselga J, et al. Trastuzumab emtansine for HER2-positive advanced breast cancer. N Engl J Med 2012;367:1783-91.

[4] Jackson DY. Processes for Constructing Homogeneous Antibody Drug Conjugates. Org Process Res Dev 2016;20:852-66.

[5] Chudasama V, Maruani A, Caddick S. Recent advances in the construction of antibody-drug conjugates. Nat Chem 2016;8:114-9.

[6] Sau S, Alsaab HO, Kashaw SK, Tatiparti K, lyer AK. Advances in antibody-drug conjugates: A new era of targeted cancer therapy. Drug Discov Today 2017;22:1547-56.

[7] Perez HL, Cardarelli PM, Deshpande S, Gangwar S, Schroeder GM, Vite GD, et al. Antibodydrug conjugates: Current status and future directions. Drug Discov Today 2014;19:869-81.

[8] LoRusso PM, Weiss D, Guardino E, Girish S, Sliwkowski MX. Trastuzumab emtansine: A unique antibody-drug conjugate in development for human epidermal growth factor receptor 2positive cancer. Clin Cancer Res 2011;17:6437-47.

[9] Lamb YN. Inotuzumab Ozogamicin: First Global Approval. Drugs 2017;77:1603-10.

[10] Godwin CD, Gale RP, Walter RB. Gemtuzumab ozogamicin in acute myeloid leukemia. Leukemia 2017;31:1855-68.

[11] Brotzel F, Mayr H. Nucleophilicities of amino acids and peptides. Org Biomol Chem 2007;5:3814-20.

[12] Moura A, Savageau MA, Alves R. Relative Amino Acid Composition Signatures of Organisms and Environments. PLoS One 2013;8.

[13] Shen BQ, Xu K, Liu L, Raab H, Bhakta S, Kenrick M, et al. Conjugation site modulates the in vivo stability and therapeutic activity of antibody-drug conjugates. Nat Biotechnol 2012;30:184-9.

[14] Baldwin AD, Kiick KL. Tunable degradation of maleimide-Thiol adducts in reducing environments. Bioconjug Chem 2011;22:1946-53.

[15] Alley SC, Benjamin DR, Jeffrey SC, Okeley NM, Meyer DL, Sanderson RJ, et al. Contribution of linker stability to the activities of anticancer immunoconjugates. Bioconjug Chem 2008;19:759-65.

[16] Tumey LN, Charati M, He T, Sousa E, Ma D, Han X, et al. Mild method for succinimide hydrolysis on ADCs: Impact on ADC potency, stability, exposure, and efficacy. Bioconjug Chem 
2014;25:1871-80.

[17] Lyon RP, Setter JR, Bovee TD, Doronina SO, Hunter JH, Anderson ME, et al. Self-hydrolyzing maleimides improve the stability and pharmacological properties of antibody-drug conjugates. Nat Biotechnol 2014;32:1059-62.

[18] Fontaine SD, Reid R, Robinson L, Ashley GW, Santi D V. Long-term stabilization of maleimidethiol conjugates. Bioconjug Chem 2015;26:145-52.

[19] Christie RJ, Fleming R, Bezabeh B, Woods R, Mao S, Harper J, et al. Stabilization of cysteinelinked antibody drug conjugates with $\mathrm{N}$-aryl maleimides. J Control Release 2015;220:660-70.

[20] Kalia D, Pawar SP, Thopate JS. Stable and Rapid Thiol Bioconjugation by Light-Triggered Thiomaleimide Ring Hydrolysis. Angew Chemie - Int Ed 2017;56:1885-9.

[21] Ponte JF, Sun X, Yoder NC, Fishkin N, Laleau R, Coccia J, et al. Understanding How the Stability of the Thiol-Maleimide Linkage Impacts the Pharmacokinetics of Lysine-Linked AntibodyMaytansinoid Conjugates. Bioconjug Chem 2016;27:1588-98.

[22] Dovgan I, Kolodych S, Koniev O, Wagner A. 2-(Maleimidomethyl)-1,3-Dioxanes (MD): A Serum-Stable Self-hydrolysable Hydrophilic Alternative to Classical Maleimide Conjugation. Sci Rep 2016;6:2-7.

[23] Tobaldi E, Dovgan I, Mosser M, Becht J-M, Wagner A. Structural investigation of cyclo-dioxo maleimide cross-linkers for acid and serum stability. Org Biomol Chem 2017:9305-10.

[24] Badescu G, Bryant P, Bird M, Henseleit K, Swierkosz J, Parekh V, et al. Bridging disulfides for stable and defined antibody drug conjugates. Bioconjug Chem 2014;25:1124-36.

[25] Bernardes GJL, Chalker JM, Errey JC, Davis BG. Facile conversion of cysteine and alkyl cysteines to dehydroalanine on protein surfaces: Versatile and switchable access to functionalized proteins. J Am Chem Soc 2008;130:5052-3.

[26] Toda N, Asano S, Barbas CF. Rapid, stable, chemoselective labeling of thiols with JuliaKocieński-like reagents: A serum-stable alternative to maleimide-based protein conjugation. Angew Chemie - Int Ed 2013;52:12592-6.

[27] Koniev O, Leriche G, Nothisen M, Remy JS, Strub JM, Schaeffer-Reiss C, et al. Selective irreversible chemical tagging of cysteine with 3-arylpropiolonitriles. Bioconjug Chem 2014;25:202-6.

[28] Smith MEB, Caspersen MB, Robinson E, Morais M, Maruani A, Nunes JPM, et al. A platform for efficient, thiol-stable conjugation to albumin's native single accessible cysteine. Org Biomol Chem 2015;13:7946-9.

[29] Kalia D, Malekar P V., Parthasarathy M. Exocyclic Olefinic Maleimides: Synthesis and Application for Stable and Thiol-Selective Bioconjugation. Angew Chemie - Int Ed 2016;55:1432-5.

[30] Gregory JD. The Stability of N-Ethylmaleimide and its Reaction with Sulfhydryl Groups. J Am Chem Soc 1955;77:3922-3.

[31] Knight P. Hydrolysis of $\mathrm{p}-\mathrm{NN}^{\prime}$-phenylenebismaleimide and its adducts with cysteine. Implications for cross-linking of proteins. Biochem J 1979;179:191-7.

[32] Forte N, Livanos M, Miranda E, Morais M, Yang X, Rajkumar VS, et al. Tuning the Hydrolytic Stability of Next Generation Maleimide Cross-Linkers Enables Access to Albumin-Antibody Fragment Conjugates and tri-scFvs. Bioconjug Chem 2018;29:486-92. 
[33] Morais M, Nunes JPM, Karu K, Forte N, Benni I, Smith MEB, et al. Optimisation of the dibromomaleimide (DBM) platform for native antibody conjugation by accelerated postconjugation hydrolysis. Org Biomol Chem 2017;15:2947-52.

[34] Chudasama V, Smith MEB, Schumacher FF, Papaioannou D, Waksman G, Baker JR, et al. Bromopyridazinedione-mediated protein and peptide bioconjugation. Chem Commun 2011;47:8781.

[35] Maruani A, Smith MEB, Miranda E, Chester KA, Chudasama V, Caddick S. A plug-and-play approach to antibody-based therapeutics via a chemoselective dual click strategy. Nat Commun 2015;6:1-9.

[36] Robinson E, Nunes JPM, Vassileva V, Maruani A, Nogueira JCF, Smith MEB, et al. Pyridazinediones deliver potent, stable, targeted and efficacious antibody-drug conjugates (ADCs) with a controlled loading of 4 drugs per antibody. RSC Adv 2017;7:9073-7.

[37] Scapin G, Yang X, Prosise WW, McCoy M, Reichert P, Johnston JM, et al. Structure of fulllength human anti-PD1 therapeutic IgG4 antibody pembrolizumab. Nat Struct Mol Biol 2015;22:953-8.

[38] Ghuman J, Zunszain PA, Petitpas I, Bhattacharya AA, Otagiri M, Curry S. Structural basis of the drug-binding specificity of human serum albumin. J Mol Biol 2005;353:38-52. 